\title{
SEPARATION OF DNA IN MICROFLUIDIC SYSTEMS
}

\author{
H. G. Craighead, J. Han and S. W. Turner \\ School of Applied and Engineering Physics, Cornell University \\ Ithaca, NY 14853
}

\begin{abstract}
It is possible to fabricate fluidic systems with dimensions comparable to the size of DNA molecules. By controlling the time and spatial dependence of electric fields one can drive, sort and separate molecules by a variety of physical mechanisms. We have created nanoconstrictions in fluidic channels that act as entropic barriers to DNA molion. We have used the size dependence of these entropic effects to separate DNA. In this paper we describe the design and fabrication two such types of devices and their performance.
\end{abstract}

\section{INTRODUCTION}

Gel electrophoresis is the standard method of separating DNA for genetic sequencing and analysis. The size-dependent electrophoretic mobility of DNA when driven through a polymeric restricting gel results in a spatial separation of DNA fragments by length. This separation appears as bands in an electropherogram. The efficiency of separation of DNA by gel electrophoresis decreases with increasing molecular length as the mobility differences become less pronounced for larger molecular sizes. We have addressed primarily this issue in the systems described here. We can design the molecular size region of interest by the choice of geometry and dimensions that are defined by lithography.

The use of microfabricated systems offers several additional possible improvements for DNA separation devices. Microfabricated systems could, for example, be designed to work with smaller amounts of material. The microfabrication approach also easily allows for parallelism so that many separations can take place simultaneously on a "chip". If gel-free systems can be used, it should be easier integration of different processes on a single chip and extraction of separated DNA should be more straightforward. Gel-free microfabricated approaches for the creation of artificial sieving structures have been suggested and met with some success. [1] Microfabricated devices that sort DNA molecules by diffusion rate have also been demonstrated. [2]

We have developed several fabrication approaches that can be integrated with optical detection and incorporate controlled geometrical constrictions in fluid channels. These approaches have enabled fabrication of structures smaller than the radius of gyration of large DNA molecules and could be used for rapid separation of the DNA.

\section{ENTROPIC TRAPPING ARRAY}

We fabricated a fluid channel with an array of narrow gaps. The cross sectional schematic of this is shown in Figure 1. The device is fabricated with two photolithography and etch steps, with the dimensions of the gap determined by an etch depth. The channel is sealed by anodically bonding a cap on the etched channels. DNA molecules are driven by electrophoresis through the series of narrow slits with a static electric field. [3] The narrow slit width is smaller than the radius of gyration of the DNA molecules.

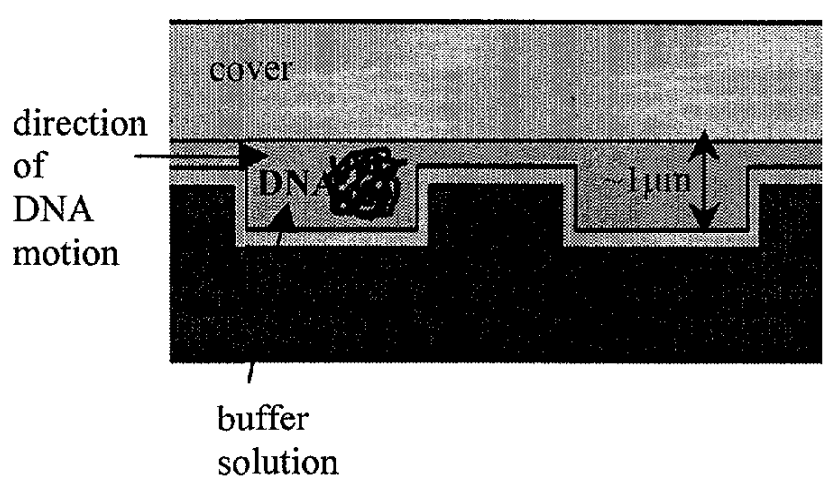

Figure 1. Cross sectional schematic of an entropic trap array.

In order for the DNA to enter the narrow region the molecule must undergo an entropically unfavorable change in conformation. The competition of the electrostatic forces driving the molecule into the narrow region with the entropic hindrance creates a free energy barrier. The probability that a molecule will surmount this barrier is proportional to the width of the molecule encountering the narrow gap. This width is approximately proportional to the square root of the number of base pairs in the molecule. [4]

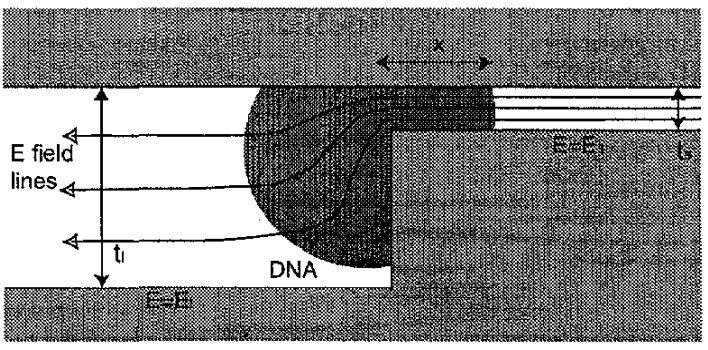

Figure 2. Schematic of the deformation and escape of a DNA molecule from a trap. 
Figure 3 shows the results of a separation of a ladder of DNA molecules with lengths from 10 kilobases to 48 kilobases. The resolution is comparable to that from the conventional pulsed field gel electrophoresis, but can be done in substantially less time. [5]

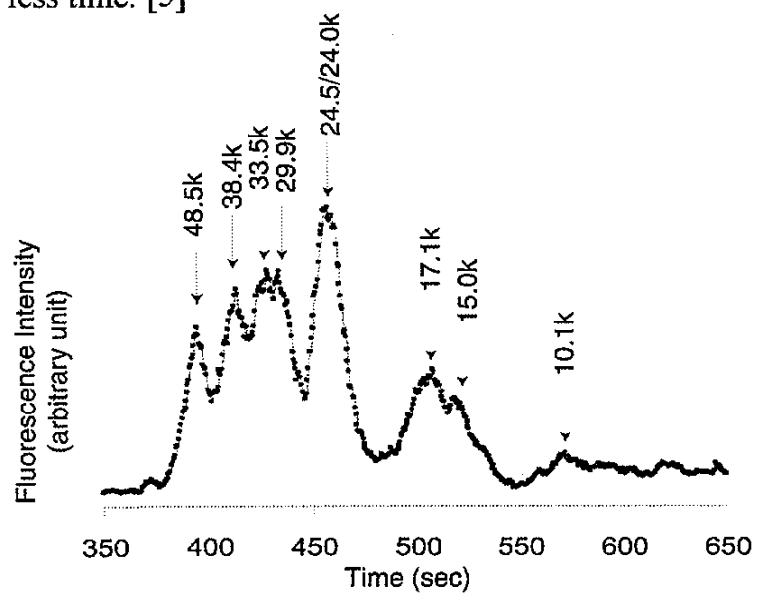

Figure 3. Separation of a DNA sample consisting of different length fragments.

This same approach should also work for other polymeric molecules.

\section{ENTROPIC RECOIL DEVICE}

We can exploit a related length-dependent trapping effect to separate DNA molecules by length. In this case we have fabricated a series of obstructions consisting of vertical columns in a thin fluid channel. This structure is formed by high-resolution lithography and sacrificial removal of a patterned layer to form the fluid system. [6] A micrograph illustrating the geometry is shown in Figure 4.

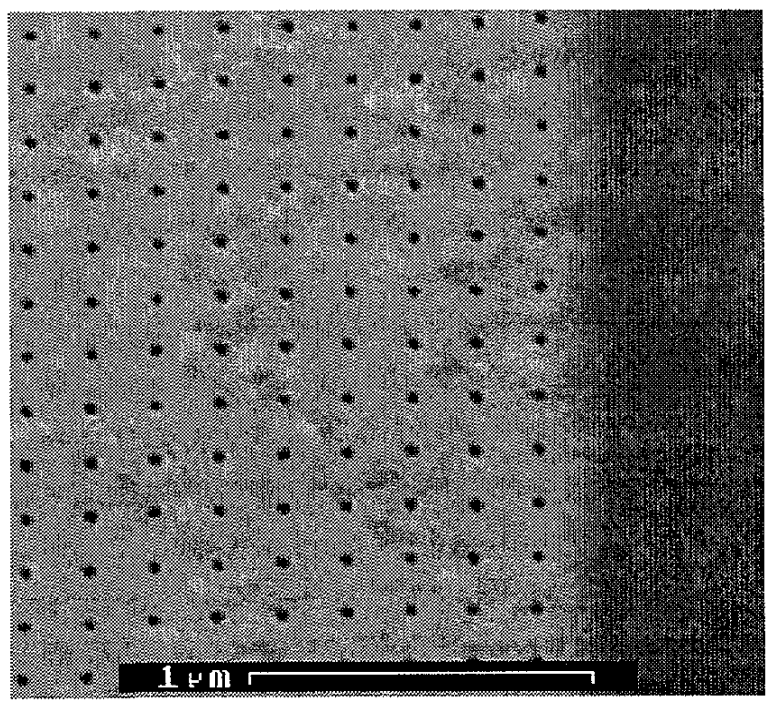

Figure 4. Electron micrograph showing a top view of entropic recoil device with the unconstrained fluid region bounded by the obstruction filled constrained region.
This structure creates an entropic trap similar to that described for the trapping array discussed above but with a different dimensionality. In this case we exploit the time difference for the passage of molecules into the constrained region. Figure 5 illustrates the basic principal of this approach.[7]

In this approach a pulsed electric field is used to drive the molecules. During the pulse molecules are driven from the entropic trap region. When the electric field is turned off molecules only partially into the constrained region will recoil and remain trapped. Longer pulse length results in the release of longer molecular lengths from the trapped area. Varying the pulse length, therefore, will result in sequential release of molecules of varying length.

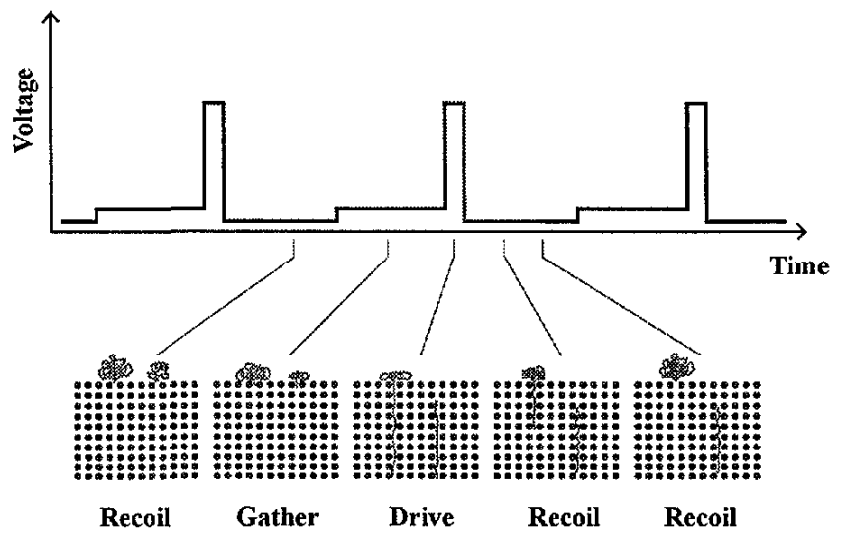

Figure 5. Schematic of the entropic recoil device finction, illustrating the behavior of a long and short DNA molecule in the time varying electric fields.

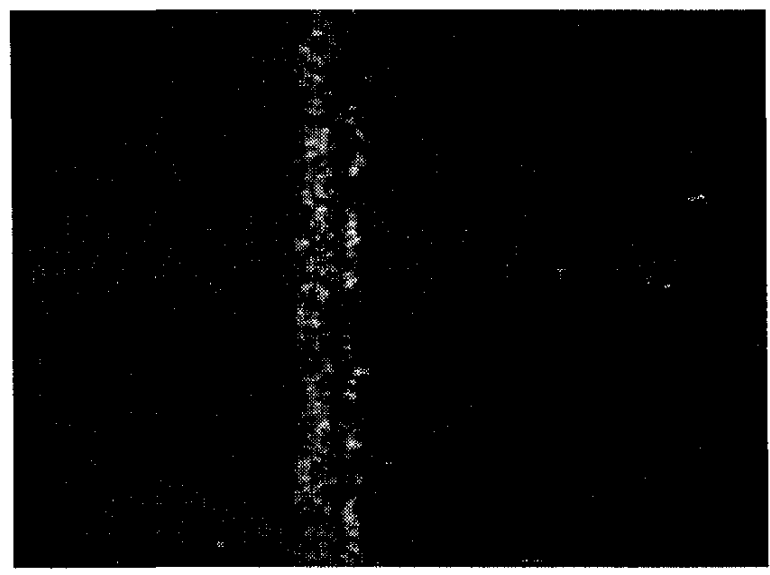

Figure 6. Fluorescence image of a band of smaller length DNA fragments released from the trapping region while the longer molecules remain trapped.

The result of such a pulsed separation is shown in Figure 6 . This fluorescence micrograph shows a band of short fluorescently labeled DNS molecules launched from the trap and traveling in the channel. These recoil structures could be staged to enhance the separation process. 


\section{CONCLUSIONS}

It is possible to rapidly sort DNA molecules by their trapping and release from nanofabricated constrictions. We have fabricated and tested scveral different systems for rapid separation of molecules in the kilobase size regime. The length region of operation should be scalable with choice of the constriction dimensions to similarly separate larger or smaller scale DNA molecular sizes. The fabrication approach with geometry controlling the device behavior allows this to be accomplished in a variety of material systems. It is also possible to integrate these fluid channels with other functional devices such as in-plane optical waveguides for integrated fluorescent excitation and detection.

\section{ACKNOWLEDGEMENTS}

This work was supported by the National Institutes of Health. Fabrication of devices was performed at the Cornell nanofabrication, supported in part by the National Science Foundation. We thank M. Cabodi for his help with the DNA recoil experiments.

\section{REFERENCES}

1. W. D. Volkmuth and R. H. Austin, Nature, 358 , 600 (1992).

2. C.F. Chou et al. Proc. Nat. Acad. Sci. 96, 13762 (1999).

3. J. Han and H. G. Craighead, "Entropic trapping and sieving of long DNA molecules in a nanofluidic channel", J. Vac. Sci. Technol A, 17, 2142 (1999).

4. J. Han, S. W. Turner and H. G. Craighead, "Entropic trapping and Escape of long DNA molecules as submicron size constrictions", Phys. Rev. Lett., 83, 1688 (1999).

5. J. Han and H. G. Craighead, "Separation of long DNA molecules in a microfabricated entropic trap array", (to appear in Science).

6. S. W. Turner, A. M. Perez, A. Lopez, and H. G. Craighead, "Monolithic nanofluidic sieving structures for DNA manipulation" J. Vac. Sci. Technol B, 16, 3835 (1998).

7. S. W. Turner, M. Cabodi and H. G. Craighead "Entropic recoil DNA separation ", APS Meeting , March 2000. 